non-equilibrium physics of many-body quantum systems. Most interestingly, it shows that many-body quantum states can survive at 'higher' temperatures for much longer than predicted by equilibrium physics. In specific non-equilibrium situations, quantum states do not undergo phase transitions as quickly (or in this case, boil as fast), as one naively expects.

In that respect, the experiment is in line with other recent studies carried out in similar systems which, for example, revealed that relaxation can be very slow in strongly correlated one-dimensional systems ${ }^{2}$, and that non-equilibrium spin structures observed in spinor condensates are very long-lived ${ }^{3}$. Such studies also enabled the observation of pre-thermalized states in quenched one-dimensional Bose gases $^{4}$, where the noise in interference patterns indicates a metastable state exhibiting a much-reduced temperature in quantum observables relevant for metrology ${ }^{5}$. All these cases demonstrate that quantum properties can persist in non-equilibrium situations for a long time, and that a quantum system might not always approach true equilibrium by a simple path.

Non-equilibrium quantum systems are prominent in many areas of physics, ranging from inflation in the early universe to the emergence of classical properties in complex quantum systems. There is a great deal more to be studied, and ultracold atomic gases provide a unique opportunity to probe the elusive physics of non-equilibrium many-body quantum systems in a multitude of different physical situations in the laboratory.

Jörg Schmiedmayer is at the Vienna Center for Quantum Science and Technology, Atominstitut, Vienna University of Technology, Stadionallee 2 1020 Vienna, Austria.

e-mail:schmiedmayer@atomchip.org

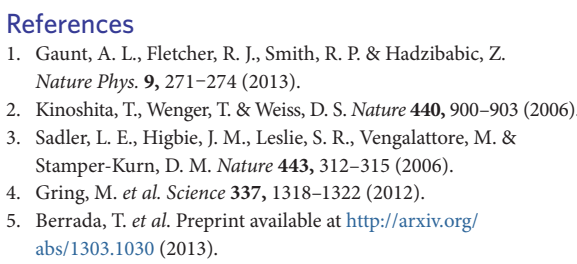

\title{
NANOTECHNOLOGY
}

\section{The can-opener effect}

Noble gas atoms are hard to tame. They seldom participate in chemical reactions, and only condense into liquids and solids at temperatures far below $0^{\circ} \mathrm{C}$. Yet Huanyao Cun and colleagues now show that argon atoms can be coaxed into regular two-dimensional lattices at room temperature (Nano Lett. http://doi.org/ 10.1021/n|400449y; 2013).

Cun et al. started with a single layer of hexagonal boron nitride ( $h-B N$ ) on top of a rhodium(111) surface. Driven by the tendency of nitrogen atoms to bind to rhodium atoms, the h-BN sheet spontaneously adopts a corrugated form to compensate for the intrinsic lattice mismatch. Scanning tunnelling microscopy shows that the wrinkled layer has a honeycomb structure - just like a flat, undeformed h-BN sheet, but with a larger lattice constant $(3.2 \mathrm{~nm})$. A closer look reveals that this $h-B N$ nanomesh consists of 'pores', where the sheet is in close contact with the substrate, surrounded by 'wires' - regions with a slightly larger sheet-substrate distance. The pores lie at the centres of hexagonal cells, the edges of which are formed by the wires, producing the honeycomb superstructure.

Exposing the nanomesh to a beam of $\mathrm{Ar}^{+}$ions results in the implantation of argon atoms between the rhodium substrate and the h-BN sheet. Using scanning tunnelling microscopy, photoemission spectroscopy and density-functional-theory calculations, the authors were able to show that the argon atoms occupy particular sites

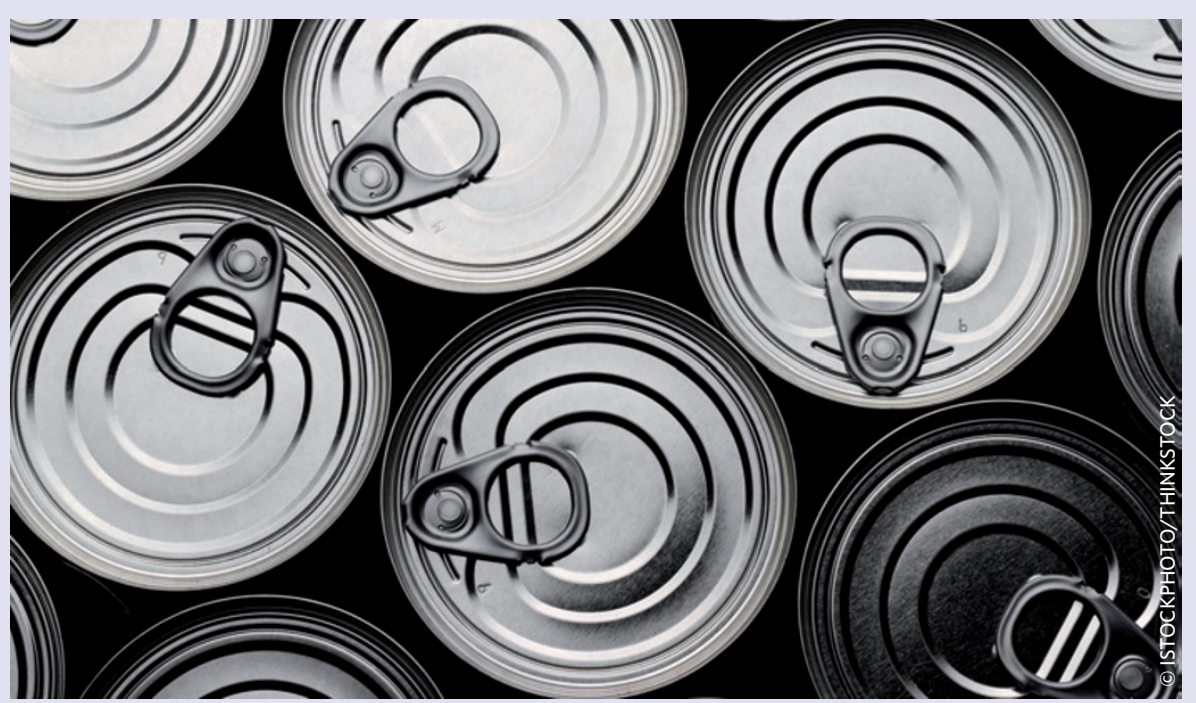

under the nanomesh - directly below wire crossings, as indicated by protrusions of the $\mathrm{h}-\mathrm{BN}$ sheet. The argon atoms thus take on an ordered two-dimensional arrangement.

Remarkable behaviour is observed when this sandwich of rhodium, argon and boron nitride is heated up to $900 \mathrm{~K}$. The argon protrusions at wire crossings disappear, and the nanomesh starts to develop holes. Pieces of h-BN appear on the nanomesh, each flake lying close to a hole, and of a similar size. Cun et al. suggest that these flakes have been cut out of the nanomesh at pore sites, similar to the way the lid is peeled off a tin can.

What they call "the can-opener effect" is a consequence of the implanted argon atoms: bombarding the nanomesh with $\mathrm{Ar}^{+}$ions kicks out boron and nitrogen atoms, leaving vacancies. These defects migrate to pore edges, where they become active sites for thermally induced bond breaking. Thus $\mathrm{h}-\mathrm{BN}$ flakes are cut from the mesh, and then diffuse away.

Cun etal. have made similar observations when neon atoms are used in place of argon, and the effect is also seen for a graphene layer on a ruthenium(0001) substrate. The holes in the nanomesh form a regular array of potential anchoring sites for molecules, hinting at possible applications in the manufacture of new metamaterials.

\section{BART VERBERCK}

